\title{
A proposed family of variationally correlated first-order density matrices for spin-polarized three-electron model atoms.
}

\author{
Ali Akbari ${ }^{1}$, Norman H. March ${ }^{2,3}$, and Angel Rubio ${ }^{1}$ \\ 1 Nano-Bio Spectroscopy Group and ETSF Scientific Development Centre, \\ Departamento de Física de Materiales, \\ Centro de Física de Materiales CSIC-UPV/EHU-MPC and DIPC, \\ Universidad del País Vasco UPV/EHU, \\ Av. Tolosa 72, E-20018 San Sebastián, Spain. \\ 2 Department of Physics, \\ University of Antwerp, Antwerp, Belgium. \\ 3 Oxford University, Oxford, England.
}

\begin{abstract}
In early work of March and Young (1959), it was pointed out for spin-free fermions that a firstorder density matrix (1DM) for $N-1$ particles could be constructed from a 2DM $(\Gamma)$ for $N$ fermions divided by the diagonal of the $1 \mathrm{DM}$, the density $n\left(\mathbf{r}_{1}\right)$, as $2 \Gamma\left(\mathbf{r}_{1}, \mathbf{r}_{2}^{\prime} ; \mathbf{r}_{1}, \mathbf{r}_{2}\right) / n\left(\mathbf{r}_{1}\right)$ for any arbitrary fixed $\mathbf{r}_{1}$. Here, we thereby set up a family of variationally valid 1 DMS constructed via the above proposal, from an exact $2 \mathrm{DM}$ we have recently obtained for four electrons in a quintet state without confining potential, but with pairwise interparticle interactions which are harmonic. As an indication of the utility of this proposal, we apply it first to the two-electron (but spin-compensated) Moshinsky atom, for which the exact 1DM can be calculated. Then the 1DM is found for spin-polarized threeelectron model atoms. The equation of motion of this correlated 1DM is exhibited and discussed, together with the correlated kinetic energy density, which is shown explicitly to be determined by the electron density.
\end{abstract}

\section{BACKGROUND AND OUTLINE}

A lot of interest is currently centering on the use of loworder density matrices (DMS) in the many-body theory of atoms, molecules and clusters. The importance of such DMS was already clear to Löwdin [1] more than half a century ago, and has been summarized by Coleman [2]. Early work of March and Young [3-5] is also highly relevant in the present context of variationally valid loworder density matrix theory.

Here we shall mainly be concerned with a few electrons $(N=2,4)$ in model atoms, though we shall also briefly refer to electron densities which transcend spherical symmetry (e.g. by applying an electric field to say the Be atom). For reasons that will become clear below, the focus of our work will be on the spin-polarized quintet and quartet states in relation to models of $\mathrm{Be}$ and $\mathrm{Li}$ atoms respectively.

Some quantitative background to the above will be set out immediately below for the reader's convenience. Following Löwdin [1], and neglecting spin, with $N$ Fermions we assume at the outset an antisymmetric wave function $\Psi\left(\mathbf{r}_{1}, \mathbf{r}_{2}, \ldots, \mathbf{r}_{N}\right)$ such that, in finite systems, it is normalized to unity.

Then the first- and second-order DMS, denoted respectively by $\gamma$ and $\Gamma$, are defined by

$\gamma\left(\mathbf{r}_{1}^{\prime}, \mathbf{r}_{1}\right)=N \int \Psi^{*}\left(\mathbf{r}_{1}^{\prime}, \mathbf{r}_{2}, \ldots, \mathbf{r}_{N}\right) \Psi\left(\mathbf{r}_{1}, \mathbf{r}_{2}, \ldots, \mathbf{r}_{N}\right) d \mathbf{r}_{2} \ldots d \mathbf{r}_{N}$ and

$$
\begin{aligned}
& \Gamma\left(\mathbf{r}_{1}^{\prime}, \mathbf{r}_{2}^{\prime} ; \mathbf{r}_{1}, \mathbf{r}_{2}\right)=\frac{N(N-1)}{2} \\
& \times \int \Psi^{*}\left(\mathbf{r}_{1}^{\prime}, \mathbf{r}_{2}^{\prime}, \mathbf{r}_{3}, \ldots, \mathbf{r}_{N}\right) \Psi\left(\mathbf{r}_{1}, \mathbf{r}_{2}, \mathbf{r}_{3}, \ldots, \mathbf{r}_{N}\right) d \mathbf{r}_{3} \ldots d \mathbf{r}_{N} .
\end{aligned}
$$

Evidently, the density $n(\mathbf{r})$ then can be found from the diagonal of the 1DM in Eq.(1). March and Young [3] next consider the function $\Phi$ defined by

$$
\Phi\left(\mathbf{r}_{2}, \ldots, \mathbf{r}_{N} ; \mathbf{r}_{1}\right)=\left(\frac{N}{n\left(\mathbf{r}_{1}\right)}\right)^{\frac{1}{2}} \Psi\left(\mathbf{r}_{1}, \mathbf{r}_{2}, \ldots, \mathbf{r}_{N}\right)
$$

which is readily shown to satisfy the normalization condition

$$
\int \Phi^{*}\left(\mathbf{r}_{2}, \ldots, \mathbf{r}_{N} ; \mathbf{r}_{1}\right) \Phi\left(\mathbf{r}_{2}, \ldots, \mathbf{r}_{N} ; \mathbf{r}_{1}\right) d \mathbf{r}_{2} \ldots d \mathbf{r}_{N}=1
$$

March and Young [3] demonstrated, because $\Phi$ as defined in Eq.(3) is antisymmetric in $\mathbf{r}_{2}, \mathbf{r}_{3}, \ldots, \mathbf{r}_{N}$, that it can be utilized to construct the DMS for $N-1$ Fermions, as follows

$$
\gamma_{N-1}\left(\mathbf{r}_{2}^{\prime}, \mathbf{r}_{2} ; \mathbf{r}_{1}\right)=\frac{2 \Gamma\left(\mathbf{r}_{1}, \mathbf{r}_{2}^{\prime} ; \mathbf{r}_{1}, \mathbf{r}_{2}\right)}{n\left(\mathbf{r}_{1}\right)}
$$

This is a $1 \mathrm{DM}$ for $N-1$ Fermions, for any arbitrary fixed $\mathbf{r}_{1}$. This result is the key to what follows, the outline of which is briefly as summarized immediately below. In section 2, a two-Fermion example is given, even though not spin polarized, in which a 1DM for one-level occupancy is presented as a rather gentle introduction to the main section 3 of the article. There use is made of the 
work of Bruch [6] in which four Fermions of spin half, mutually interacting with pairwise attractive harmonic interactions, and in the quintet state, are considered. Bruch's achievement was to calculate the spatial wave function exactly for this four Fermion spin-polarized state with translational invariance. The March and Young (MY) proposal is then used in section 3 to calculate a 1DM for three spin-polarized spin half Fermions in therefore a quartet state. Section 4 constitutes a summary, together with proposals for future work which should prove fruitful.

Thus, we turn immediately to our first quite simple example: the two-Fermion Moshinsky model with both harmonic confinement and pairwise harmonic forces.

\section{SPIN-COMPENSATED DENSITY MATRICES: TEST OF MARCH-YOUNG (MY) PROPOSAL ON THE MOSHINSKY MODEL}

Using the two-electron Moshinsky atom with external potential $V_{\text {ext }}(r)=\frac{1}{2} k r^{2}$ and interparticle interaction $u\left(r_{12}\right)=\frac{1}{2} K r_{12}^{2}$, one of us [7] has earlier given the correlated $1 \mathrm{DM}$ for $k=1$. Generalizing away from this value to a general $k$, the spatial ground-state spin-compensated wave function has the general shape

$$
\Psi\left(\mathbf{r}_{1}, \mathbf{r}_{2}\right)=C e^{-C_{1}\left(r_{1}^{2}+r_{2}^{2}\right)} e^{-C_{2} \mathbf{r}_{1} \cdot \mathbf{r}_{2}}
$$

where $C, C_{1}$ and $C_{2}$ are known in terms of $k$ and $K$ force constants. These relations are summarized in Eq.(A1) of the Appendix. The corresponding 2DM in the desired form to test the MY proposal in [3], given also below in Eq.(9) is evidently

$$
\Gamma\left(\mathbf{r}_{1}, \mathbf{r}_{2}^{\prime} ; \mathbf{r}_{1}, \mathbf{r}_{2}\right)=C^{2} e^{-C_{1}\left(r_{2}^{2}+r_{2}^{\prime 2}+2 r_{1}^{2}\right)} e^{-C_{2} \mathbf{r}_{1} \cdot\left(\mathbf{r}_{2}^{\prime}+\mathbf{r}_{2}\right)} .
$$

Multiplying by two and integrating over $\mathbf{r}_{1}$ we get the $1 \mathrm{DM} \gamma\left(\mathbf{r}_{2}^{\prime}, \mathbf{r}_{2}\right)$ as

$$
\gamma\left(\mathbf{r}_{2}^{\prime}, \mathbf{r}_{2}\right)=2\left(\frac{\pi}{2 C_{1}}\right)^{\frac{3}{2}} C^{2} e^{-C_{1}\left(r_{2}^{2}+r_{2}^{\prime 2}\right)+\frac{C_{2}^{2}}{8 C_{1}}\left|\mathbf{r}_{2}+\mathbf{r}_{2}^{\prime}\right|^{2}}
$$

Evidently therefore $n\left(\mathbf{r}_{1}\right)$ needed in Eq.(5) is simply a Gaussian $e^{\left(-2 C_{1}+C_{2}^{2} / 2 C_{1}\right) r_{1}^{2}}$ multiplied by a constant for normalization of the Fermion density to two electrons.

Hence, for one-particle, assuming the unproved (for spin-compensated case used here) MY result, Eq.(5), the $1 \mathrm{DM}$ for $N-1=1$ reads

$$
\gamma_{1}\left(\mathbf{r}_{2}^{\prime}, \mathbf{r}_{2} ; \mathbf{r}_{1}\right)=\frac{2 \Gamma\left(\mathbf{r}_{1}, \mathbf{r}_{2}^{\prime} ; \mathbf{r}_{1}, \mathbf{r}_{2}\right)}{n\left(\mathbf{r}_{1}\right)}
$$

and from Eqs.(7)-(9) we find

$$
\gamma_{1}\left(\mathbf{r}_{2}^{\prime}, \mathbf{r}_{2} ; \mathbf{r}_{1}\right)=d e^{-C_{1}\left(r_{2}^{2}+r_{2}^{\prime 2}\right)} e^{-C_{2} \mathbf{r}_{1} \cdot\left(\mathbf{r}_{2}^{\prime}+\mathbf{r}_{2}\right)}
$$

where

$$
d=\left(\frac{2 C_{1}}{\pi}\right)^{\frac{3}{2}} e^{\left(-C_{2}^{2} / 2 C_{1}\right) r_{1}^{2}}
$$

is a constant which depends on $\mathbf{r}_{1}$. Hence the density $n_{1}$ is given by $n_{1}\left(\mathbf{r}_{2} ; \mathbf{r}_{1}\right)$ as

$$
n_{1}\left(\mathbf{r}_{2} ; \mathbf{r}_{1}\right)=d e^{-2 C_{1} r_{2}^{2}} e^{-2 C_{2} \mathbf{r}_{1} \cdot \mathbf{r}_{2}} .
$$

Given the MY relation as valid in this case, for fixed arbitrary $\mathbf{r}_{1}$, the result (12) must satisfy the von Weizsäcker equation for one-level occupation as

$$
\nabla_{\mathbf{r}_{2}}^{2} n^{\frac{1}{2}}\left(\mathbf{r}_{2} ; \mathbf{r}_{1}\right)+\frac{2 m}{\hbar^{2}}\left(\epsilon-V\left(\mathbf{r}_{2} ; \mathbf{r}_{1}\right)\right) n^{\frac{1}{2}}\left(\mathbf{r}_{2} ; \mathbf{r}_{1}\right)=0
$$

for arbitrary fixed $\mathbf{r}_{1}$ and also we note that $\epsilon$ depends on this quantity. Hence, to within the energy $\epsilon$ appearing in Eq.(13), the Slater-Kohn-Sham one-body potential $V\left(\mathbf{r}_{2} ; \mathbf{r}_{1}\right)$ can be extracted. This, in general, is not spherically symmetric in $\mathbf{r}_{2}$, because of the appearance of the scalar product $\mathbf{r}_{1} \cdot \mathbf{r}_{2}$ in the exponent in Eq.(12). The result is given by

$$
\frac{2 m}{\hbar^{2}} V\left(\mathbf{r}_{2} ; \mathbf{r}_{1}\right)=4 C_{1}^{2} r_{2}^{2}+4 C_{1} C_{2} \mathbf{r}_{1} \cdot \mathbf{r}_{2} \text {. }
$$

Of course, for $\mathbf{r}_{1}$ (or $C_{2}$ ) set equal to zero we have simply harmonic confinement.

We shall come back to generaliztion of the von Weizsäcker Eq.(13) in section 3, to which we now turn.

\section{A VARIATIONALLY VALID 1DM FOR THREE PARTICLE LI-LIKE QUARTET STATES.}

We return to the major focus of this article: namely to use the MY result in Eq.(9) in a proven case of complete spin polarization. Thus, the present authors have recently calculated the exact correlated 2DM for the quintet state of the four-electron Moshinsky atom [9], but with the confining potential switched off. Then, Eq.(5), for $(\mathrm{N}-1=3)$, reads

$$
\gamma_{3}\left(\mathbf{r}_{2}^{\prime}, \mathbf{r}_{2} ; \mathbf{r}_{1}\right) \propto \Gamma\left(\mathbf{r}_{1}, \mathbf{r}_{2}^{\prime} ; \mathbf{r}_{1}, \mathbf{r}_{2}\right)
$$

since the "density" $n\left(\mathbf{r}_{1}\right)=\gamma\left(\mathbf{r}_{1}, \mathbf{r}_{1}\right)$ is itself simply a constant because of the translational invariance of the starting Hamiltonian. The 2DM $\Gamma$ is given by [9] $(\omega=$ $\left.\sqrt{\frac{K}{m}}\right)$ as

$$
\begin{aligned}
\Gamma\left(\mathbf{r}_{1}^{\prime}, \mathbf{r}_{2}^{\prime} ; \mathbf{r}_{1}, \mathbf{r}_{2}\right) \propto & \left\{\left(\frac{4 \hbar}{m \omega}-\left|\mathbf{R}-\mathbf{R}^{\prime}\right|^{2}\right) \mathbf{r} \cdot \mathbf{r}^{\prime}\right. \\
& +(\mathbf{R} \cdot \mathbf{r})\left(\mathbf{R} \cdot \mathbf{r}^{\prime}\right)+\left(\mathbf{R}^{\prime} \cdot \mathbf{r}^{\prime}\right)\left(\mathbf{R}^{\prime} \cdot \mathbf{r}\right) \\
& \left.-\left(\mathbf{R}^{\prime} \cdot \mathbf{r}\right)\left(\mathbf{R} \cdot \mathbf{r}^{\prime}\right)-(\mathbf{R} \cdot \mathbf{r})\left(\mathbf{R}^{\prime} \cdot \mathbf{r}^{\prime}\right)\right\} \\
& \times e^{-\frac{m \omega}{4 \hbar}\left(\left|\mathbf{R}-\mathbf{R}^{\prime}\right|^{2}+\mathbf{r}^{2}+\mathbf{r}^{\prime 2}\right)}
\end{aligned}
$$

$\mathbf{r}$ and $\mathbf{R}$ being defined (the primed coordinates are defined in the same way but with primes instead) by

$$
\mathbf{R}=\frac{\mathbf{r}_{1}+\mathbf{r}_{2}}{2}, \quad \mathbf{r}=\mathbf{r}_{1}-\mathbf{r}_{2}
$$


The density $n_{3}\left(\mathbf{r}_{2} ; \mathbf{r}_{1}\right)$, which is normalized to three Fermions, can be calculated with the pair density $\Gamma\left(\mathbf{r}_{1}, \mathbf{r}_{2} ; \mathbf{r}_{1}, \mathbf{r}_{2}\right)$. This gives $n_{3}\left(\mathbf{r}_{2} ; \mathbf{r}_{1}\right)$ as

$$
n_{3}\left(\mathbf{r}_{2} ; \mathbf{r}_{1}\right)=\frac{2}{\pi^{\frac{3}{2}}}\left(\frac{m \omega}{2 \hbar}\right)^{\frac{5}{2}} r^{2} e^{-\frac{m \omega}{2 \hbar} r^{2}}
$$

or equivalently

$$
\begin{aligned}
n_{3}\left(\mathbf{r}_{2} ; \mathbf{r}_{1}\right)= & \frac{2}{\pi^{\frac{3}{2}}}\left(\frac{m \omega}{2 \hbar}\right)^{\frac{5}{2}}\left(r_{2}^{2}+\left(r_{1}^{2}-2 \mathbf{r}_{1} \cdot \mathbf{r}_{2}\right)\right) \\
& \times e^{-\frac{m \omega}{2 \hbar}\left(r_{1}^{2}-2 \mathbf{r}_{1} \cdot \mathbf{r}_{2}\right)} e^{-\frac{m \omega}{2 \hbar} r_{2}^{2}}
\end{aligned}
$$

Evidently, the simplest choice $\mathbf{r}_{1}=0$ yields

$$
n_{3}\left(\mathbf{r}_{2} ; \mathbf{r}_{1}=0\right)=\frac{2}{\pi^{\frac{3}{2}}}\left(\frac{m \omega}{2 \hbar}\right)^{\frac{5}{2}} r_{2}^{2} e^{-\frac{m \omega}{2 \hbar} r_{2}^{2}}
$$

which is, of course a spherical electron density. But for all other choices of the vector $\mathbf{r}_{1}$, the density $n_{3}\left(\mathbf{r}_{2} ; \mathbf{r}_{1}\right)$ in Eq.(19) becomes non-spherical. Again, as proposed in the two-electron (singlet) case in section 2, one can get a family of spherical correlated electron densities for three spin-polarized Fermions by averaging over the angle between $\mathbf{r}_{2}$ and $\mathbf{r}_{1}$ in Eq.(19).

Before turning to the off-diagonal form $\gamma_{3}\left(\mathbf{r}_{2}^{\prime}, \mathbf{r}_{2} ; \mathbf{r}_{1}\right)$ of Eq.(19), let us use its diagonal element $n_{3}\left(\mathbf{r}_{2} ; \mathbf{r}_{1}\right)$ to extract a "bosonization" generalization of the argument in section 2 based on Eq.(13). Since we are now dealing with three parallel spin electrons, we must generalize the one-body potential $V\left(\mathbf{r}_{2} ; \mathbf{r}_{1}\right)$ entering the equivalent of Eq.(13) to include the Pauli potential $[10,11] V_{p}\left(\mathbf{r}_{2} ; \mathbf{r}_{1}\right)$ : i.e. the total potential in Eq.(13) becomes $V+V_{p}$. Hence we can immediately write, to within an additive "constant", now involving $\mathbf{r}_{1}$ of course, the generalization of Eq.(13) as

$$
\frac{2 m}{\hbar^{2}}\left(V\left(\mathbf{r}_{2} ; \mathbf{r}_{1}\right)+V_{p}\left(\mathbf{r}_{2} ; \mathbf{r}_{1}\right)\right)=\frac{\nabla_{\mathbf{r}_{2}}^{2} n_{3}^{\frac{1}{2}}\left(\mathbf{r}_{2} ; \mathbf{r}_{1}\right)}{n_{3}^{\frac{1}{2}}\left(\mathbf{r}_{2} ; \mathbf{r}_{1}\right)}
$$

But from Eq.(18)

$$
n_{3}^{\frac{1}{2}}\left(\mathbf{r}_{2} ; \mathbf{r}_{1}\right)=\frac{\sqrt{2}}{\pi^{\frac{3}{4}}}\left(\frac{m \omega}{2 \hbar}\right)^{\frac{5}{4}} r e^{-\frac{m \omega}{4 \hbar} r^{2}}
$$

where $r=\left|\mathbf{r}_{2}-\mathbf{r}_{1}\right|$. Hence, we find the explicit form

$$
\frac{2 m}{\hbar^{2}}\left(V\left(\mathbf{r}_{2} ; \mathbf{r}_{1}\right)+V_{p}\left(\mathbf{r}_{2} ; \mathbf{r}_{1}\right)\right)=\frac{2}{r^{2}}+\left(\frac{m \omega}{2 \hbar}\right)^{2} r^{2}
$$

It is relevant to note from DFT [12] that (see also [11])

$$
V_{p}=\frac{\delta T_{s}}{\delta n}-\frac{\delta T_{W}}{\delta n}
$$

$T_{s}$ and $T_{W}$ being the single-particle and von Weizsäcker kinetic energy respectively. $\frac{\delta T_{W}}{\delta n}$ is given explicitly by $[12]$

$$
\frac{m}{\hbar^{2}} \frac{\delta T_{W}}{\delta n}=\frac{1}{8}\left(\frac{\nabla n}{n}\right)^{2}-\frac{1}{4} \frac{\nabla^{2} n}{n}
$$

We return now to the off-diagonal 1DM $\gamma_{3}$ for three spinpolarized Fermions given in Eq.(15), when the right hand side is obtained from Eq.(16) by putting $\mathbf{r}_{1}^{\prime}=\mathbf{r}_{1}$. The proportionality constant is found from the fact that its diagonal is given in Eq.(18). Hence, we have

$$
\begin{aligned}
\gamma_{3}\left(\mathbf{r}_{2}^{\prime}, \mathbf{r}_{2} ; \mathbf{r}_{1}\right)= & \frac{1}{\pi^{\frac{3}{2}}}\left(\frac{m \omega}{2 \hbar}\right)^{\frac{7}{2}}\left\{\left(\frac{4 \hbar}{m \omega}-\left|\mathbf{R}-\mathbf{R}^{\prime}\right|^{2}\right) \mathbf{r} \cdot \mathbf{r}^{\prime}\right. \\
& +(\mathbf{R} \cdot \mathbf{r})\left(\mathbf{R} \cdot \mathbf{r}^{\prime}\right)+\left(\mathbf{R}^{\prime} \cdot \mathbf{r}^{\prime}\right)\left(\mathbf{R}^{\prime} \cdot \mathbf{r}\right) \\
& \left.-\left(\mathbf{R}^{\prime} \cdot \mathbf{r}\right)\left(\mathbf{R} \cdot \mathbf{r}^{\prime}\right)-(\mathbf{R} \cdot \mathbf{r})\left(\mathbf{R}^{\prime} \cdot \mathbf{r}^{\prime}\right)\right\} \\
& \times e^{-\frac{m \omega}{4 \hbar}\left(\left|\mathbf{R}-\mathbf{R}^{\prime}\right|^{2}+\mathbf{r}^{2}+\mathbf{r}^{\prime 2}\right)}
\end{aligned}
$$

Evidently, the correlated kinetic energy $t_{3}\left(\mathbf{r}_{2} ; \mathbf{r}_{1}\right)$ for arbitrary fixed $\mathbf{r}_{1}$ is then to be found from

$$
\begin{aligned}
t_{3}\left(\mathbf{r}_{2} ; \mathbf{r}_{1}\right) & =-\left.\frac{\hbar^{2}}{2 m} \nabla_{\mathbf{r}_{2}}^{2} \gamma_{3}\left(\mathbf{r}_{2}^{\prime}, \mathbf{r}_{2} ; \mathbf{r}_{1}\right)\right|_{\mathbf{r}_{2}^{\prime}=\mathbf{r}_{2}} \\
& =\frac{\hbar \omega}{4 \pi^{\frac{3}{2}}}\left(\frac{m \omega}{2 \hbar}\right)^{\frac{5}{2}}\left(\frac{25}{2} r^{2}-\frac{m \omega}{\hbar} r^{4}\right) e^{-\frac{m \omega}{2 \hbar} r^{2}}
\end{aligned}
$$

But utilizing Eq.(18), Eq.(27) can be rewritten in terms of $n_{3}\left(\mathbf{r}_{2} ; \mathbf{r}_{1}\right)$ as below:

$$
t_{3}\left(\mathbf{r}_{2} ; \mathbf{r}_{1}\right)=\frac{\hbar \omega}{8}\left(\frac{25}{2}-\frac{m \omega}{\hbar} r^{2}\right) n_{3}\left(\mathbf{r}_{2} ; \mathbf{r}_{1}\right)
$$

To proceed further, it can be found from Eq.(18) that

$$
\left(2-\frac{m \omega}{\hbar} r^{2}\right) \frac{\mathbf{r}}{r^{2}}=\frac{\nabla n_{3}}{n_{3}}
$$

and from it, these relations follow:

$$
\hbar \omega\left(4-\frac{m \omega}{\hbar} r^{2}\right)=\frac{\hbar^{2}}{m}\left(\frac{4}{r^{2}}-\frac{\left|\nabla n_{3}\right|^{2}}{n_{3}^{2}}\right)
$$

and

$$
\hbar \omega=\frac{\hbar^{2}}{m r^{2}}\left(2-\frac{\nabla n_{3} \cdot \mathbf{r}}{n_{3}}\right) .
$$

Now, by using Eq.(30) and Eq.(31), finally Eq.(28) becomes

$$
t_{3}\left(\mathbf{r}_{2} ; \mathbf{r}_{1}\right)=\frac{\hbar^{2}}{8 m}\left(\frac{21}{r^{2}} n_{3}-\frac{17}{2} \frac{\nabla n_{3} \cdot \mathbf{r}}{r^{2}}-\frac{\left|\nabla n_{3}\right|^{2}}{n_{3}}\right)
$$

Fig. 2 shows the form of Eq.(27) for $t_{3}$ compared with $n_{3}$ in Eq.(18). The total kinetic energy can be calculated by integration from Eq.(27) and it yields

$$
T=\int t_{3}\left(\mathbf{r}_{2} ; \mathbf{r}_{1}\right) d \mathbf{r}_{2}=\frac{45}{16} \hbar \omega .
$$

To complete this section, we turn to the equation of motion of the correlated 1DM $\gamma_{3}$. Amovilli and March [13] have expanded $\gamma_{3}$ in a complete set of Slater-KohnSham orbitals $\psi_{i}(\mathbf{r})$, which can be taken as real in the 

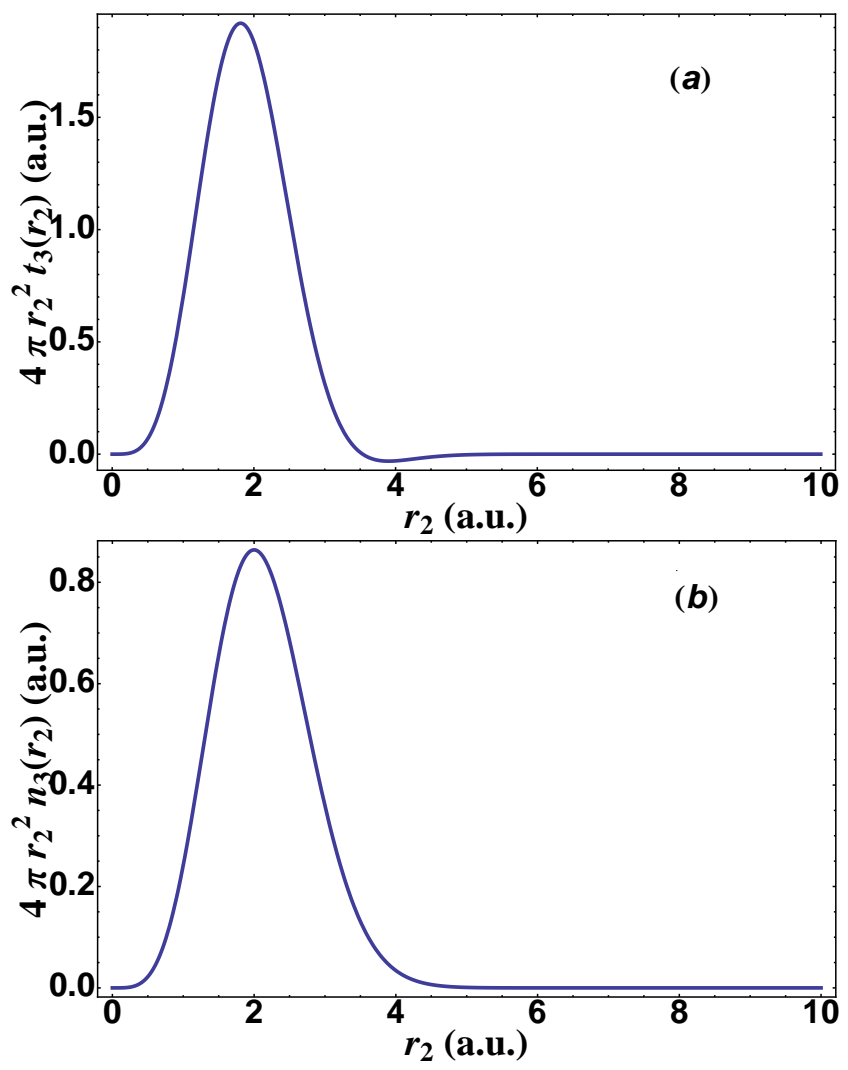

FIG. 1. (a) plot of the correlated kinetic energy density versus $r_{2}$ for three particles as in Eq.(27). (b) plot for the correlated density in Eq.(18). Here, $\mathbf{r}_{1}$ is set equal to zero and $\omega=1$.

absence of a magnetic field. In [13], it is then demonstrated that $\gamma_{3}$ satisfies the partial differential equation of motion

$$
\begin{aligned}
& \left(\nabla_{\mathbf{r}_{2}}^{2}-\nabla_{\mathbf{r}_{2}^{\prime}}^{2}\right) \gamma_{3}\left(\mathbf{r}_{2}^{\prime}, \mathbf{r}_{2} ; \mathbf{r}_{1}\right)= \\
& \frac{2 m}{\hbar^{2}}\left(V\left(\mathbf{r}_{2} ; \mathbf{r}_{1}\right)-V\left(\mathbf{r}_{2}^{\prime} ; \mathbf{r}_{1}\right)+\Delta\left(\mathbf{r}_{2}, \mathbf{r}_{2}^{\prime} ; \mathbf{r}_{1}\right)\right) \gamma_{3}\left(\mathbf{r}_{2}^{\prime}, \mathbf{r}_{2} ; \mathbf{r}_{1}\right)
\end{aligned}
$$

The potential $V(\mathbf{r})$ is the DFT one-body potential which generates, by definition the Slater-Kohn-Sham orbitals. In Eq.(34), $\Delta$ is written in [13] in terms of the coefficients $n_{i j}$ entering the expansion of $\gamma_{3}$ in Slater-Kohn-Sham orbitals $\psi_{i}(\mathbf{r})$, the eigenvalues $\epsilon_{i}$ being involved through the difference $\epsilon_{i}-\epsilon_{j}$. But our interest here is in the one-body potential $V(\mathbf{r})$, which already enters Eq.(23), but added to the Pauli potential $V_{p}(\mathbf{r})$. Our aim below is to obtain both $V(\mathbf{r})$ and $V_{p}(\mathbf{r})$ for the above model. Using $\gamma_{3}$ in Eq.(34) gives

$$
V\left(\mathbf{r}_{2} ; \mathbf{r}_{1}\right)=\frac{3}{16} m \omega^{2} r^{2}
$$

This result comes directly from the explicit form of
Eq.(34), namely

$$
\begin{aligned}
& \left(\nabla_{\mathbf{r}_{2}}^{2}-\nabla_{\mathbf{r}_{2}^{\prime}}^{2}\right) \gamma_{3}\left(\mathbf{r}_{2}^{\prime}, \mathbf{r}_{2} ; \mathbf{r}_{1}\right)= \\
& \left(\frac{3 m^{2} \omega^{2}}{8 \hbar^{2}}\right)\left(\left|\mathbf{r}_{2}-\mathbf{r}_{1}\right|^{2}-\left|\mathbf{r}_{2}^{\prime}-\mathbf{r}_{1}\right|^{2}\right) \gamma_{3}\left(\mathbf{r}_{2}, \mathbf{r}_{2}^{\prime} ; \mathbf{r}_{1}\right) \\
& +\left(\frac{m^{2} \omega^{2}}{32 \hbar^{2}}\right) \mathbf{r} \cdot \mathbf{r}^{\prime}\left(\mathbf{r}+\mathbf{r}^{\prime}\right) \cdot\left(\mathbf{r}_{2}-\mathbf{r}_{2}^{\prime}\right) e^{-\frac{m \omega}{4 \hbar}\left(\frac{\left|\mathbf{r}_{2}-\mathbf{r}_{2}^{\prime}\right|^{2}}{4}+r^{2}+r^{\prime 2}\right)} .
\end{aligned}
$$

The first term on the left hand side of Eq.(36) can readily be seen to yield Eq.(35). The final term in this model as set out in [13] involves off-diagonal occupations number plus differences in one-electron eigenvalues, as referred to already below Eq.(34). Hence from Eq.(23) we can calculate the Pauli potential $V_{p}$. The important term at small $r$ is $\frac{3 \hbar^{2}}{8 m r^{2}}$. The sum of $V$ and $V_{p}$ at large $r$ is already clear from Eq.(23) as proportional to $r^{2}$. But from DFT, $V$ plus $V_{p}$ entering Eq.(23) is a functional of the density $n_{3}$, just as for $t_{3}$.

\section{SUMMARY AND FUTURE DIRECTIONS}

The aim of the present study has been to examine some consequences for the 1DM of the MY proposal in Eq.(5), (see Eq.(9) for $N=2$ and also the end of section 1 for an arbitrary number $N$ of Fermions). Two potentially important cases have been discussed. The more profound of these is to display a correlated 1DMS for the three-electron spin-polarized systems, having both nonspherical and spherical Fermion densities on the diagonal. In the non-spherical case (e.g. appropriate when an electric field is switched on to an initially spherical model atom) the equation of motion of the correlated 1DM is derived, following the study of Amovilli and March [13]. In the simpler example of the two-electron Moshinsky atom , the MY proposal has led us to families of both spherical and non-spherical densities. For the non-spherical case, the one-body potential leading to the one-level density $n_{1}$ is attractively compact, and is given in Eq.(14). Also Eq.(12) is used in section II to derive the one-body potential set out in Eq.(A3), see also Fig.(2). But the result to be especially emphasized is the correlated kinetic energy density $t_{3}$ in Eq.(27) which is shown in Eq.(32) to be expressible solely in terms of the density $n_{3}$ plus $\nabla n_{3}$. The variationally valid three-Fermions $1 \mathrm{DM} \gamma_{3}$ leads to the kinetic energy $T[n, \nabla n]$ given in Eq.(32). Performing the functional derivative in the case of spherical symmetry $\left(\mathbf{r}_{1}=0\right)$ yields

$$
\begin{aligned}
\frac{\delta T}{\delta n(r)} & =\frac{21 \hbar^{2}}{8 m r^{2}}-\frac{17}{2} \frac{\delta}{\delta n(r)} \int \frac{\nabla n_{3} \cdot \mathbf{r}}{r^{2}} d \mathbf{r}-\frac{\delta T_{W}}{\delta n(r)} \\
& =\frac{59 \hbar^{2}}{16 m r^{2}}-\frac{\delta T_{W}}{\delta n(r)}
\end{aligned}
$$

which is, though for a special model, relevant for the theorems of DFT. 


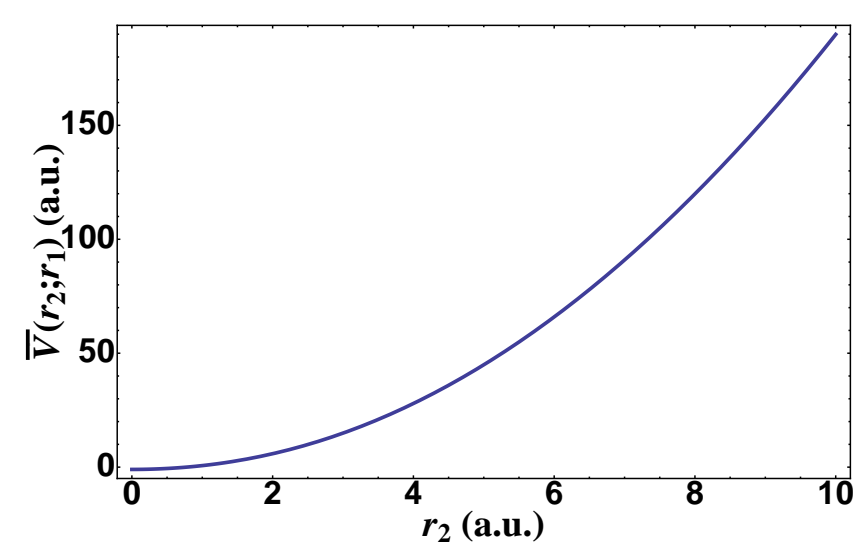

FIG. 2. Calculated one-body spherical potential $\bar{V}\left(\mathbf{r}_{2} ; \mathbf{r}_{1}\right)$ from Eq.(A3), with $C_{2} r_{1}$ and $C_{1}$ equal to one.

As to the future, it will be worthwhile to attempt the generalization of the present proposal to treat spincompensated states and also partial spin-polarization for general $N$. Then, the further exact wave functions with translational-invariance given by Bruch [6] for Fermions with pairwise harmonic attractions for $N=4$ should lead to new interacting correlated 1DMS for three electrons, which may well have interesting practical applications in atomic physics as well as in quantum chemistry.

\section{ACKNOWLEDGMENT}

N. H. March (NHM) wishes to acknowledge the major contributions made to his understanding of the general area in which this study lies by Professors W. H. Young and C. Amovilli over some decades. Most generous hospitality of Professor P. M. Echenique at DIPC has made possible NHM's contribution to the present study. Also his continuing affiliation with the University of Antwerp (UA) is due to support from Professors D. Lamoen and C. Van Alsenoy through BOF-NOI(UA). We acknowledge financial support from the European Research Council Advanced Grant DYNamo (ERC-2010-AdG, Proposal No. 267374), Spanish Grants No. FIS2011-65702-C02-01 and No. PIB2010US- 00652, ACI-Promociona (Grant No. ACI2009-1036), Grupos Consolidados UPV/EHU del Gobierno Vasco (Grant No. IT-319-07), Consolider
nanoTHERM (Grant No. CSD2010-00044), Fondo Social Europeo (FSE) and the Spanish Research Council (CSIC) through their Junta para la Ampliación de Estudios fellowship (JAE-Predoc-2008).

\section{Appendix A: Some technical details relating to section 2}

The Moshinsky wave function in Eq.(6) is written completely in terms of two quantity $C_{1}$ and $C_{2}$ and the normalization constant $C$. These are given below explicitly in terms of the force constant $k$ of the external potential and $K$ for the interparticle interaction $u\left(r_{12}\right)$ :

$$
\begin{aligned}
& C=\frac{\beta^{\frac{3}{2}}}{2 \sqrt{2} \pi^{\frac{3}{2}} a^{3}}, \quad C_{1}=\frac{1+\beta^{2}}{8 a^{2}}, \quad C_{2}=\frac{1-\beta^{2}}{4 a^{2}}, \\
& a=\left(\frac{\hbar}{2 \sqrt{m k}}\right)^{\frac{1}{2}}, \quad \beta=\left(\frac{2 K+k}{k}\right)^{\frac{1}{4}}
\end{aligned}
$$

Next, We turn to the density $n_{1}$ in Eq.(12). We summarize below a spherical family of one-level electron densities and the corresponding one-body potential. This family is constructed explicitly by multiplying Eq.(12) by volume element $d \Omega$ and integrate over the angle between $\mathbf{r}_{1}$ and $\mathbf{r}_{2}$ to find

$$
\bar{n}_{1}\left(r_{2} ; r_{1}\right)=\frac{d}{C_{2} r_{1} r_{2}}\left(e^{C_{2} r_{1} r_{2}}-e^{-C_{2} r_{1} r_{2}}\right) e^{-2 C_{1} r_{2}^{2}}
$$

With this density $\bar{n}_{1}$ inserted for $n$ in the von Weizsäcker Eq.(13) we extract the one-body potential $\bar{V}\left(\mathbf{r}_{2} ; \mathbf{r}_{1}\right)$ to within an additive constant, as

$$
\begin{aligned}
\frac{2 m}{\hbar^{2}} \bar{V}\left(\mathbf{r}_{2} ; \mathbf{r}_{1}\right) & =-\frac{1}{4 r_{2}^{2}}+4 C_{1}^{2} r_{2}^{2}-\left(\frac{C_{2} r_{1}}{2}\right)^{2} \operatorname{coth}^{2}\left(C_{2} r_{1} r_{2}\right) \\
& +\left(\frac{C_{2} r_{1}}{2 r_{2}}-2 C_{1} C_{2} r_{1} r_{2}\right) \operatorname{coth}\left(C_{2} r_{1} r_{2}\right)
\end{aligned}
$$

This means that the Schrödinger equation can be solved exactly for this potential $\bar{V}\left(\mathbf{r}_{2} ; \mathbf{r}_{1}\right)$ for arbitrary $\mathbf{r}_{1}$, the corresponding wave function $\bar{\Psi}$ being simply the square root of the right hand side of Eq.(A2). Fig.2 depicts the general shape of the potential in Eq.(A2) for a particular choice of $C_{2} r_{1}$ and $C_{1}$.
[1] P. O. Löwdin, Phys. Rev. 97, 1474 (1955).

[2] A. J. Coleman, Rev. Mod. Phys. 35668 (1963).

[3] N. H. March and W. H. Young, Phil. Mag. 4, 384 (1959).

[4] W. H. Young and N. H. March, Proc. Roy. Soc. (London) A256, 62 (1960).

[5] See also N. H. March and G. G. N. Angilella. Many-Body Theory of Molecules, Clusters and Condensed Phases (World Scientific, Singapore, 2010).

[6] L. W. Bruch, J. Chem. Phys. 72, 5511 (1980).
[7] N. H. March, Phys. Lett. A 30663 (2002).

[8] C. Amovilli and N. H. March, Phys. Rev. A, 83044502 (2011).

[9] A. Akbari, C. Amovilli, N. H. March and A. Rubio, Chemical Physics Letters, 536,162 (2012).

[10] M. Levy and A. Gorling, Phil. Mag., B69 763 (1994).

[11] See also N. H. March, J. Mol. Structure (Theochem), 943 77 (2010).

[12] R. G. Parr and W. Yang, Density Functional Theory 
of Atoms and Molecules (Oxford University Press: New York 1989).

(2011).

[13] C. Amovilli and N. H. March; in course of publication 